\title{
epsilon-Support Vector and Large-Scale Data Mining Problems*
}

\author{
Gang $\mathrm{Kou}^{1,2}$, Yi Peng ${ }^{2, * *}$, Yong Shi ${ }^{2,3}$, and Zhengxin $\mathrm{Chen}^{2}$ \\ ${ }^{1}$ Thomson Co., R\&D, 610 Opperman Drive, Eagan, MN 55123, USA \\ ${ }^{2}$ College of Information Science \& Technology, University of Nebraska at Omaha, Omaha, NE \\ 68182, USA \\ \{gkou, ypeng, yshi, zchen\}@mail.unomaha.edu \\ ${ }^{3}$ Chinese Academy of Sciences Research Center on Data Technology \& Knowledge Economy, \\ Graduate University of the Chinese Academy of Sciences, 100080, China \\ Tel.: ++1-402-4030269
}

\begin{abstract}
Data mining and knowledge discovery has made great progress during the last fifteen years. As one of the major tasks of data mining, classification has wide business and scientific applications. Among a variety of proposed methods, mathematical programming based approaches have been proven to be excellent in terms of classification accuracy, robustness, and efficiency. However, there are several difficult issues. Two of these issues are of particular interest of this research. The first issue is that it is challenging to find optimal solution for large-scale dataset in mathematical programming problems due to the computational complexity. The second issue is that many mathematical programming problems require specialized codes or programs such as CPLEX or LINGO. The objective of this study is to propose solutions for these two problems. This paper proposed and applied mathematical programming model to classification problems to address two aspects of data mining algorithm: speed and scalability.
\end{abstract}

Keywords: Data mining, Classification, Mathematical Programming, Multiple criteria decision making, Support Vector Machine.

\section{Introduction}

Over the years, optimization-based algorithms have shown their effectiveness in data mining classification (e.g., Bugera, Konno, and Uryasev, 2002) and Mathematical programming (MP) based algorithm is one of the optimization-based classification algorithms (e.g., Kou et al 2006). Nevertheless, due to the limitation of computation power and memory, it is difficult to apply the MP algorithm, or similar optimization algorithms, to huge datasets which may contain millions of observations. As the size

\footnotetext{
* This research has been partially supported by grants \#70621001, \#70531040,\#70472074, National Natural Science Foundation of China; 973 Project \#2004CB720103, Ministry of Science and Technology, China; and BHP Billiton Co., Australia.

*** The corresponding author.
} 
of today's databases is continuously increasing, it is highly important that data mining algorithms are able to perform their functions regardless of the sizes of datasets.

Develop mining algorithms that scale to real-life massive databases is the first research challenges proposed by Bradley, Fayyad, and Mangasarian in their overview of applying mathematical programming for data mining. They also pointed out that "approaches that assume that data can fit in main memory need to be revised or redesigned (Bradley, Fayyad, and Mangasarian 1998)." MP based algorithm is such an approach that requires the data to fit in main memory. This requirement comes from the fact that constraint matrix must be loaded into main memory in order to achieve an acceptable computation time and the size of constraint matrix is determined by the size of the training dataset. Therefore, as the size of dataset increases, the computation time increases and performance degraded.

The objectives of this research are: (1) to propose a new epsilon-support vector approach, and (2) to apply this approach to large scale data mining problem and analyze substantial datasets (size of $\mathrm{O}\left(10^{9}\right)$ ). The results indicate that this new approach has the potential to handle arbitrary-size of datasets.

This paper is organized in three parts. The next section describes the epsilonsupport vector for Multi-criteria Convex Quadratic programming (MCQP) two-group classification model. Section 3 presents the experimental results. The last section concludes the paper.

\section{Epsilon-Support Vector (eSV) Approach}

Each row of a $n \times r$ matrix $\boldsymbol{A}=\left(\boldsymbol{A}_{1}, \ldots, \boldsymbol{A}_{n}\right)^{\mathrm{T}}$ is an vector $\boldsymbol{A}_{i}$ $=\left(a_{i 1}, \ldots, a_{i r}\right) \in \mathfrak{R}^{r}$ which corresponds to one of the records in the training dataset of a binary classification problem, $i=1, \ldots, n ; n$ is the total number of records in the dataset. Two groups, $G_{1}$ and $G_{2}$, are predefined while $G_{1} \cap G_{2}=\Phi$ and $A_{i}$ $\in\left\{G_{1} \cup G_{2}\right\}$. A boundary scalar $b$ can be selected to separate $G_{1}$ and $G_{2}$. Let $\boldsymbol{X}=$ $\left(x_{1}, \ldots, x_{r}\right)^{T} \in \mathfrak{R}^{r}$ be a vector of real number to be determined. In the classification problem, $\boldsymbol{A}_{\boldsymbol{i}} \boldsymbol{X}$ is the score for the $i^{\text {th }}$ data record. If all records are linear separable and an element $\boldsymbol{A}_{\boldsymbol{i}}$ is correctly classified, then let $\beta_{i}$ be the distance from $\boldsymbol{A}_{i}$ to $b$, and consider the linear system, $\boldsymbol{A}_{i} \boldsymbol{X}=b-\beta_{i}, \forall \boldsymbol{A}_{i} \in G_{1}$ and $\boldsymbol{A}_{i} \boldsymbol{X}=b+\beta_{i}, \forall \boldsymbol{A}_{\boldsymbol{i}} \in$ $G_{2}$. However, if we consider the case where the two groups are not linear separable because of mislabeled records, a "Soft Margin" and slack distance variable $\alpha_{i}$ need to be introduced. Previous equations now transforms to $\boldsymbol{A}_{i} \boldsymbol{X}=b+\alpha_{i}-\beta_{i}, \forall \boldsymbol{A}_{i} \in$ $G_{1}$ and $\boldsymbol{A}_{i} \boldsymbol{X}=b-\alpha_{i}+\beta_{i}, \forall \boldsymbol{A}_{i} \in G_{2}$. To complete the definitions of $\beta_{i}$ and $\alpha_{i}$, let $\beta_{i}=0$ for all misclassified elements and $\alpha_{i}$ equals to zero for all correctly classified elements. Incorporating the definitions of $\beta_{i}$ and $\alpha_{i}$, out classification models could be set as: 
Minimize $\frac{1}{2}\|X\|_{2}^{2}+W_{\alpha} \sum_{i=1}^{n} \alpha_{i}^{2}-W_{\beta} \sum_{i=1}^{n} \beta_{i}+\frac{W_{b}}{2} b^{2}$

Subject to: $\boldsymbol{Y}(<\boldsymbol{A} \cdot \boldsymbol{X}>-\mathrm{eb})=\delta^{\prime} e-\alpha+\beta$

where $\boldsymbol{Y}$ is a given $n \times n$ diagonal matrix, $e=(1,1, \ldots, 1)^{\mathrm{T}}, \alpha=\left(\alpha_{1}, \ldots, \alpha_{n}\right)^{T}$, $\beta=\left(\beta_{1}, \ldots, \beta_{n}\right)^{T}, X$ and $b$ are unrestricted.

The Lagrange function corresponding to Model 3 is $L(X, b, \eta, \theta)=\frac{1}{2}\|X\|_{2}^{2}+\frac{W_{\alpha}}{2} \sum_{i=1}^{n} \eta_{i}^{2}+W_{\beta} \sum_{i=1}^{n} \eta_{i}+\frac{W_{b}}{2} b^{2}-\theta^{\mathrm{T}}(\mathrm{Y}(<\mathrm{A} \cdot \mathrm{X}>-\mathrm{eb})-\mathrm{e} \delta+\eta)$ where $\theta=\left(\theta_{1}, \ldots, \theta_{n}\right)^{T}, \eta=\left(\eta_{1}, \ldots, \eta_{n}\right)^{T}, \theta_{i}, \eta_{i} \in \mathfrak{R}$.

According to Wolfe Dual Theorem, $\nabla_{X} L(X, b, \eta, \theta)=X-A^{T} Y \theta=0$, $\nabla_{b} L(X, b, \eta, \theta)=W_{b} b+e^{T} Y \theta=0, \nabla_{\eta} L(X, b, \eta, \theta)=W_{\alpha} \eta+W_{\beta} e-\theta=0$.

Introduce the above 3 equations to the constraints of Model 3 , we can get:

$$
\begin{gathered}
Y\left(\left(A \cdot A^{T}\right) Y \theta+\frac{1}{W_{b}} e\left(e^{T} Y \theta\right)\right)+\frac{1}{W_{\alpha}}\left(\theta-W_{\beta} e\right)=\delta^{\prime} e \\
\Rightarrow \theta=\frac{\left(\delta^{\prime}+\frac{W_{\beta}}{W_{\alpha}}\right) e}{\frac{I}{W_{\alpha}}+Y\left(\left(A \cdot A^{T}\right)+\frac{1}{W_{b}} e e^{T}\right) Y}
\end{gathered}
$$

In algorithm, we describe how to use this model in a classification problem.

\section{Algorithm}

Input: a $n \times r$ matrix $\boldsymbol{A}$ as the training dataset, a $n \times n$ diagonal matrix $\boldsymbol{Y}$ labels the class of each record.

Output: classification accuracies for each group in the training dataset, score for every record, decision function $\left(\left(X^{*} \cdot A_{i}\right)-b^{*}\right)\left\{\begin{array}{l}>0, \Rightarrow A_{i} \in G_{1} \\ \leq 0, \Rightarrow A_{i} \in G_{2}\end{array}\right.$

Step 1. compute $\theta^{*}=\left(\theta_{1}, \ldots, \theta_{n}\right)^{T}$ by (5). $W_{\beta}, W_{\alpha}, W_{b}$ are chosen by cross validation.

Step 2. compute $X^{*}=A^{T} Y \theta^{*}, b^{*}=\frac{-1}{W_{b}} e^{T} Y \theta^{*}$.

Step 3. classify a incoming $A_{i}$ by using decision function $\left(\left(X^{*} \cdot A_{i}\right)-b^{*}\right)\left\{\begin{array}{l}>0, \Rightarrow A_{i} \in G_{1} \\ \leq 0, \Rightarrow A_{i} \in G_{2}\end{array}\right.$

END 
In existing SVM approaches (Boser et al 1992), the sparsity of the optimal $\alpha_{i}^{*}$ (many of $\left.\alpha_{i}^{*}=0\right)$ is the key in solving large scale SVM problem and the support vectors $\left(\alpha_{i}^{*}=0\right)$ are the points at the hyperplane. Similarly, the support vector $\boldsymbol{A}_{i}$ of our models can be defined as $\alpha_{i}^{*}=\beta_{i}^{*}=0$. Due to the formulation of the model objectives, almost none of the data points $A_{i}$ lie on two adjusted bounding hyper planes $b \pm 1$. Thus most of the time $\alpha_{i}^{*}$ and $\beta_{i}^{*}$ do not equal to zero simultaneously. A Karush-Kuhn-Tucker (KKT) condition that has been used in previous support vector approaches (Cristianini and Shawe-Taylor 2000, Schölkopf, and Smola 2002) can not be held any more because of the introduction of distance variable $\beta_{i}$ in our model. As a result, we need to establish another condition for those data points $A_{i}$ whose corresponding $\left|\alpha_{i}^{*}-\beta_{i}^{*}\right|<\mathcal{E}$ to define the epsilon-support vectors (eSVs) such that the computation complexity can be reduced for large scale problems. $\mathcal{E}$ is picked so that only a small part (e.g. 1\%) of the records belongs to epsilon-support vectors. Generally, if $n<1000000$, Model 2 can use the whole dataset; otherwise, a sample training set (e.g. $1 \%$ of the population) can be randomly generated from the original data. Using the sample set, we can find an optimal solution and epsilon-support vectors for the original dataset. All epsilon-support vectors are used to re-train the model repeatedly until the classification results meet certain criteria/threshold. A stratified random sampling approach is incorporated into our algorithm for large scale data mining problem $\left(>\mathrm{O}\left(10^{9}\right)\right)$.

\section{Stratified Random Sampling}

Since MP based algorithm requires training datasets to fit in main memory, the size of training dataset is limited by the capacity of main memory $\left(\mathrm{O}\left(10^{6 \sim 8}\right)\right)$. One possible solution is to use only part of the training dataset when the dataset size is extremely huge $\left(>\mathrm{O}\left(10^{9}\right)\right)$. However, this approach may loss valuable information that exists in the unused part of the training dataset. In order to make the best use of the training dataset, we employ a revised stratified random sampling.

Let's briefly describe how standard stratified random sampling works. First, the dataset is partitioned into groups of data called strata. Each data belongs to one and only one stratum. Second, a sample is selected by some design within each stratum (Thompson 1992). As a sampling technique, the goal of stratified random sampling is to select a portion of a population that can be used as a "representation" of the population as a whole.

\section{Algorithm}

The following procedure summarized the whole process:

\section{Algorithm 1}

Input: The training dataset $\mathrm{A}$ as the population, $\mathrm{n}$ is the number of observation in the population and is a huge number; $\mathrm{m}$ is the number of 
subpopulations which can be fitted into main memory; The testing dataset A'; Algorithm Stop criteria.

Output: Average classification accuracies; decision scores for all observations; decision function.

Step 1: A is evenly partitioned into $m$ subpopulations or strata by random selection.

Step 2: One random sample is drawn from each of $m$ subpopulations and formulate the first training set Tr.

Step 3: Compute $\theta^{*}=\left(\theta_{1}, \ldots, \theta_{n}\right)^{T}$ using $\operatorname{Tr}$ as input. $W_{\beta}, W_{\alpha}, W_{b}$ are chosen by cross validation.

Step 4: Compute $X^{*}=A^{T} Y \theta^{*}, b^{*}=\frac{-1}{W_{b}} e^{T} Y \theta^{*}$. If the performance measure meet pre-set criteria (accuracy, total computation time, etc) and the iteration times $>10$, stop; otherwise go to step 5 .

Step 5: Compute $\left|\alpha_{i}^{*}-\beta_{i}^{*}\right|$ for all observations in A.

Step 6: Suppose we have $\mathrm{n}_{1}$ observations (epsilon-support vectors (eSVs)) corresponding $\left|\alpha_{i}^{*}-\beta_{i}^{*}\right|<\mathcal{E}$ and $\mathrm{n}_{2}$ observations (Non epsilonsupport vectors) which $\left|\alpha_{i}^{*}-\beta_{i}^{*}\right|>\varepsilon$. Formulate a new set with all $\mathrm{n}_{1}$ eSVs and some $\left(\mathrm{n}_{3}, \mathrm{n}_{3} \leq \mathrm{n}_{2}\right.$ and $\left.\mathrm{n}_{3} \cong 10 \% * \mathrm{n}_{1}\right)$ non eSVs. If $\left(\mathrm{n}_{3}+\mathrm{n}_{1}\right)$ $\leq \mathrm{m}$, the new set is the new training set, otherwise, random sample $\mathrm{m}$ observations from the new set to formulate a train set. Go back to step 3.

\section{END}

* Iteration times must be greater than 10 to minimize the influence of the selection of initial training dataset.

\section{Experimental Results}

The purpose of this research is to test the applicability of our algorithm in large scale data mining problem $\left(>\mathrm{O}\left(10^{9}\right)\right)$, which is too large to be loaded into a typical PC memory $(512 \mathrm{MB}-4 \mathrm{~Gb})$ but can be saved in harddisk (40Gb-1Tb). Because we can't find any real life data in such size, we use a Normally Distributed Clustered program (NDC) (Musicant 1998) to generate datasets. Normally distributed cluster is a data generator. NDC generates data points clustered to a series of random centers and all points are multivariate normal distributed. All variables are integers for simplicity. Then, NDC randomly generates a separating plane and assign a class for each random center according to the random separating plane. NDC changes dataset separability by changing the variances of the distributions. The percentage of separability is calculated by counting how many points end up on the right and wrong side of the separating plane. NDC will provide the percentage of linear separability. 
The experimental study has two parts. The first one is to test the capability of the proposed algorithm in massive problem with low noises. The second test is to evaluate the performance of the proposed algorithm under high noises, large dataset.

\section{Massive Dataset}

The first experiment dataset consists of 100 million records and each record has 20 variables. It is balanced with $50 \%$ of the data in the first group and $50 \%$ of the data in the second group. The dataset is $95 \%$ linear separable (Musicant 1998).

\section{Algorithm 2}

Input: $\quad$ The input data set of 100 million records; Assume that at most 1 million records can be fitted into main memory.

Output: 10 -fold cross validation average classification accuracies; computing time.

Step 1: The input data set is evenly partitioned into 10 subsets by stratified random sampling selection.

Step 2: $\quad$ A standard 10-fold cross-validation set of 10 sets of training dataset and testing dataset is stored by using one of the 10 subsets as the testing dataset and using the remaining 9 subsets as training dataset. Repeat the following steps for each of the 10 sets of training dataset and testing dataset

Step 3: $\quad n=100$ million and $m=1$ million. Stop criteria: Prediction Accuracy $>$ $94.8 \%$ or computing time $>1000$ minutes. Apply algorithm 1 .

\section{END}

Table 1. Massive dataset computing results

\begin{tabular}{|r|r|r|c|r|r|}
\hline \multirow{2}{*}{ 10-fold } & \multicolumn{2}{|c|}{ training } & time & \multicolumn{2}{c|}{ testing } \\
\cline { 2 - 6 } & Class 1 & Class 2 & (seconds) & Class 1 & Class 2 \\
\hline fold 1 & $94.93 \%$ & $94.87 \%$ & 631 & $94.91 \%$ & $94.82 \%$ \\
\hline fold 2 & $94.96 \%$ & $94.82 \%$ & 933 & $94.93 \%$ & $94.82 \%$ \\
\hline fold 3 & $94.85 \%$ & $94.93 \%$ & 378 & $94.87 \%$ & $94.91 \%$ \\
\hline fold 4 & $94.88 \%$ & $94.89 \%$ & 785 & $94.87 \%$ & $94.90 \%$ \\
\hline fold 5 & $94.84 \%$ & $94.95 \%$ & 655 & $94.82 \%$ & $94.93 \%$ \\
\hline fold 6 & $94.95 \%$ & $94.93 \%$ & 1217 & $94.89 \%$ & $94.91 \%$ \\
\hline fold 7 & $94.93 \%$ & $94.93 \%$ & 467 & $94.91 \%$ & $94.86 \%$ \\
\hline fold 8 & $94.98 \%$ & $94.85 \%$ & 632 & $94.92 \%$ & $94.84 \%$ \\
\hline fold 9 & $94.87 \%$ & $94.94 \%$ & 876 & $94.85 \%$ & $94.83 \%$ \\
\hline fold 10 & $94.83 \%$ & $94.88 \%$ & 539 & $94.84 \%$ & $94.86 \%$ \\
\hline average & $94.90 \%$ & $94.90 \%$ & 711.3 & $94.88 \%$ & $94.87 \%$ \\
\hline
\end{tabular}

\section{Large Dataset with High Noise}

The first experiment dataset consists of 2 million records and each record has 10 variables. It is balanced with $50 \%$ of the data in the first group and $50 \%$ of the data in the second group. The dataset is $75 \%$ linear separable. 


\section{Algorithm 3}

Input: The input data set of 2 million records; Assume that all records can be fitted into main memory.

Output: 10-fold cross validation average classification accuracies; computing time.

Step 1: The input data set is evenly partitioned into 10 subsets by stratified random sampling selection.

Step 2: A standard 10-fold cross-validation set of 10 sets of training dataset and testing dataset is stored by using one of the 10 subsets as the testing dataset and using the remaining 9 subsets as training dataset. Repeat the following steps for each of the 10 sets of training dataset and testing dataset

Step 3: $\mathrm{n}=2$ million and $\mathrm{m}=2$ million. Stop criteria: Prediction Accuracy $>$ $70 \%$ or computing time $>1000$ minutes. Apply algorithm 1 .

\section{END}

Table 2. High noise dataset computing results

\begin{tabular}{|r|c|c|c|c|c|}
\hline \multirow{2}{*}{ 10-fold } & \multicolumn{2}{|c|}{ training } & time & \multicolumn{2}{c|}{ testing } \\
\cline { 2 - 6 } & Class 1 & Class 2 & (seconds) & Class 1 & Class 2 \\
\hline fold 1 & $71.34 \%$ & $74.33 \%$ & 1279 & $70.25 \%$ & $71.12 \%$ \\
\hline fold 2 & $72.77 \%$ & $74.13 \%$ & 849 & $72.85 \%$ & $72.47 \%$ \\
\hline fold 3 & $74.79 \%$ & $72.44 \%$ & 1537 & $72.31 \%$ & $71.23 \%$ \\
\hline fold 4 & $70.94 \%$ & $73.58 \%$ & 2574 & $70.32 \%$ & $72.09 \%$ \\
\hline fold 5 & $74.11 \%$ & $72.35 \%$ & 742 & $72.57 \%$ & $71.32 \%$ \\
\hline fold 6 & $73.56 \%$ & $74.33 \%$ & 1380 & $73.24 \%$ & $72.67 \%$ \\
\hline fold 7 & $71.90 \%$ & $70.38 \%$ & 3546 & $70.39 \%$ & $70.12 \%$ \\
\hline fold 8 & $73.89 \%$ & $72.48 \%$ & 836 & $72.37 \%$ & $72.23 \%$ \\
\hline fold 9 & $72.37 \%$ & $73.38 \%$ & 711 & $71.56 \%$ & $71.73 \%$ \\
\hline fold 10 & $73.56 \%$ & $72.84 \%$ & 1207 & $72.40 \%$ & $72.19 \%$ \\
\hline average & $72.92 \%$ & $73.02 \%$ & 1466.1 & $71.83 \%$ & $71.72 \%$ \\
\hline
\end{tabular}

\section{Conclusion}

In this research, we proposed a new epsilon-support vector approach and conducted 2 experimental studies using computer generated large scale classification problem to address two aspects of data mining algorithm: speed and scalability. The experimental results indicate that the new approach is capable in solving large scale data mining problems $\left(>\mathrm{O}\left(10^{9}\right)\right)$ and highly efficient (computing time complexity $\left.\mathrm{O}\left(\mathrm{n}^{1.5 \sim 2}\right)\right)$. This approach also has good performance when the dataset is highly noised (noises are larger than $25 \%$ of the whole data).

\section{References}

1. Boser, B. E., Guyon, I., Vapnik., V. N. : A training algorithm for optimal margin classifiers. Proceedings of the Fifth Annual Workshop on Computational Learning Theory, 5:, (1992) 144--152 
2. Bradley, P.: Mathematical Programming Approaches to Machine Learning and Data Mining. PhD thesis, University of Wisconsin, Computer Sciences Department, Madison, WI, USA, TR-98-11. (1998)

3. Bugera, V., Konno, H., Uryasev, S.: Credit Cards Scoring with Quadratic Utility Function. Journal of Multi-Criteria Decision Analysis 11 (2002) 197-211.

4. Cristianini, N. and J. Shawe-Taylor. An Introduction to Support Vector Machines. Cambridge University Press, Cambridge, UK, (2000)

5. Musicant, D. R. NDC: normally distributed clustered datasets, www.cs.wisc.edu/_musicant/data/ndc/. (1998)

6. Kou, G., Peng, Y., Shi, Y., Wise, M., Xu, W.: Using Multi-objective Linear Programming to Classify Credit Cardholder Behavior, Optimization Methods and Software, Vol. 18, (2003) 453-473

7. Kou, G., Peng, Y., Shi, Y., Wise, M., Xu, W.: Discovering Credit Cardholders' Behavior by Multiple Criteria Linear Programming, Annals of Operations Research 135 (1):, JAN (2005) 261-274

8. Schölkopf, B., Smola., A. J.: Learning with Kernels. MIT Press, (2002)

9. Smola, A., Bartlett, P., Schölkopf, B., Schuurmans, D. (eds).: Advances in Large Margin Classifiers. MIT Press, Cambridge, MA, (2000)

10. Thompson, Sampling, S.K., :A Wiley-Interscience Publication, New York. (1992)

11. Vapnik, V. N.,: The Nature of Statistical Learning Theory, Springer, New York. (1995) 